\title{
Diagnóstico accidental de Hepatitis B crónica. A propósito de dos casos
}

\author{
Dora Montiel, Ruth Peralta, Enrique Ortiz \\ Departamento de Medicina Interna. Hospital Nacional de Itaugua, Asunción-Paraguay
}

Cómo referenciar este artículo/

How to reference this article:

\begin{abstract}
Montiel D, Peralta $R$, Ortiz E. Diagnóstico accidental de Hepatitis B crónica. A propósito de dos casos. Mem. Inst. Investig. Cienc. Salud. $2016 ; 14(3): 126-130$
\end{abstract}

\section{R E S U M E N}

Se reporta el diagnóstico accidental de dos casos de hepatitis B crónica por probable transmisión vertical. El primer caso es una gestante de 32 años que es derivada al hospital para monitoreo fetal por oligoamnios; durante su internación entra en trabajo de parto, y es asistida por una profesional sin usar guantes y se produce un importante contacto con sangre. Se realiza a la paciente un control serológico revelando ser portadora de $\mathrm{HBsAg}$ pero negativa para $\mathrm{HBeAg}$; positiva para anti-HBe Ac y HBcIgG, negativa para anti HBs y $\mathrm{HBC}$ Ac IgM. El segundo caso es un paciente portador de Lupus Eritematoso Sistémico bajo tratamiento inmunosupresor, con antecedentes de tener una madre fallecida y dos hermanos portadores de VHB. La serología reveló: HBsAg (+) con carga viral de 113 copias, HBeAg $(-)$, anti-HBe $(+)$, HBcIgG $(+)$, HBcIgM $(-)$, hepatitis $C(-)$. El paciente recibió tratamiento con tenofovir. Ambos pacientes fueron diagnosticados en forma accidental y considerados ser portadores de hepatitis B crónica inactiva por probable trasmisión vertical por los antecedentes familiares. Estos dos casos constituyen una llamada de atención sobre la transmisión vertical del virus y la importancia de realizar el despistaje en la embarazada en el primer control prenatal.

Palabras clave: Hepatitis B crónica, transmisión vertical, detección accidental, portadores asintomáticos de HBsAg

\section{Incidental diagnosis of chronic hepatitis B. Report of two cases}

\begin{abstract}
A B S T R A C T
Two cases of incidentally diagnosed chronic hepatitis B of probable vertical transmission are reported. The first case is a 32 year-old pregnant patient referred to our center for fetal monitoring due to oligohydramnios; during her hospitalization the patient went into labor, the professional assisted her without gloves and there was an important contact with blood. Serological tests were performed to the patientrevealing to be a HBsAg carrier, but negative for $\mathrm{HBeAg}$, positive for anti-HBe Ac and HBcIgG, negative for anti HBs and HBC Ac IgM. The second case is a patient with Systemic Lupus Erythematosus under immunosuppressive treatment, with a history of having a deceased mother and two siblings with HBV infection. Serology revealedHBsAg (+) with viral load of 113 copies, HBeAg (-), hepatitis C (-), HBcIgG $(+)$, HBcIgM $(-)$, anti-HBe $(+)$. The patient received treatment with ten of ovir. Both patients were incidentally diagnosed and considered to be chronic inactive hepatitis $B$ carriers due to probable vertical transmission based on their family history. These two cases constitute a wake-up call about the vertical transmission of the virus and the importance of performing the screening in all pregnant women in the first prenatal care.
\end{abstract}

Key Words: Chronic hepatitis B, vertical transmission, incidentally detection asymptomatic HBsAg carriers 


\section{INTRODUCCIÓN}

Actualmente la infección por el virus de la hepatitis $B(\mathrm{VHB})$ y $\mathrm{C}(\mathrm{VHC})$ constituye un problema de salud púbica de trascendencia mundial principalmente debido a que son la causa más común de cirrosis hepática y hepatocarcinoma(1).A pesar de contarse con una vacuna eficaz contra la hepatitis $B$, la infección por este virus sigue siendo una importante causa de morbilidad y mortalidad, sobre todo en países en vía de desarrollo (1-2-3).

La infección por el VHB es diagnosticada por la detección en sangre del antígeno de superficie del VHB (HBsAg, siglas del inglés: Hepatitis Bs Antigen), el cual corresponde al marcador de tamizaje. Adicionalmente, otros marcadores serológicos y virológicos como detección de anticuerpos contra la proteína core del VHB tipo inmunoglubulina M (anti$\mathrm{HBcIgM}$ ) e inmunoglobulina G (anti-HBcIgG), anti- HBs (anticuerpos contra el HBsAg), antígeno e (HBeAg, del inglés hepatitis B e Antigen), ADN viral (carga viral) y niveles elevados de aminotransferasas (ALTs) son importantes para la clasificación del tipo de infección $(2,4,5)$.

Luego de la exposición al virus se considera que hay un periodo de incubación de 1-6 meses. La demostración de infección aguda coincide con la detección del HBsAg y de antiHBcIgM. Otros marcadores como el HBeAg, títulos elevados del genoma viral (carga viral) y niveles altos de aminotransferasas (ALTs) pueden ser detectados (1-2-3).

La infección aguda puede ser sintomática o asintomática siendo esta última una enfermedad subclínica que no es generalmente diagnosticada $(6,7)$.

La resolución clínica de la infección se caracteriza por el descenso de la carga viral a valores negativos o no detectables, producción de anti-HBe, normalización de los niveles de ALTs y seroconversión de HBsAg a anti-HBs. Debido a la persistencia del genoma del VHB en los hepatocitos, actualmente se considera que el marcadoranti-HBs no es marcador del aclaramiento viral. Por lo anterior individuos con resolución clínica pueden eventualmente desarrollar infección crónica $(4,5)$.

Aunque en la mayoría de los casos la evolución de la hepatitis aguda por virus B es favorable, con resolución espontánea del cuadro clínico en 4-8 semanas, el $90 \%$ de los casos en la infancia y el $5-10 \%$ en los adultos progresan a hepatitis crónica. Una vez establecida la infección crónica, los dos principales escenarios que llevan a la muerte de los pacientes son la cirrosis y el cáncer hepatocelular $(7,8)$.

La infección crónica por el VHB es, sin embargo, un proceso dinámico con un amplio espectro de afectaciones, en un extremo se encuentra el paciente con el antígeno $\mathrm{HBsAg}$ positivo asintomático detectado accidentalmente (IDAHS), sin evidencia clínica de enfermedad hepática y en el otro extremo se tiene la cirrosis en los estadios finales de la infección y el carcinoma hepatocelular (HCC) (3-8).

Este subgrupo de pacientes en general consultan debido a que el antígeno de superficie de la hepatitis B (HBsAg) se detecta durante un chequeo de rutina, donación de sangre, antecedentes familiares de infección por el VHB, contactos de pacientes con portadores crónicos relacionados con VHB o por presentar un hepatograma anormal. Una gran proporción de pacientes con (IDAHS) adquieren la infección a través de la exposición perinatal o por transmisión vertical. Aunque en la presentación son asintomáticos y saludables, una gran proporción de IDAHS muestran un hallazgo histológico anormal en la biopsia hepática y conlleva el riesgo de progresión a cirrosis y el cáncer de hígado, relacionados con los niveles de ALT y el estado sérico de HBeAg (3).

En Paraguay, se desconoce la prevalencia de portadores crónicos de la infección por el virus de la Hepatitis B a pesar de ser una infección de notificación obligatoria(9)por el subregistro de los casos notificados. En América Latina, Paraguay se ubica entre los países deendemicidadbaja,0,5 al $2 \%$ de seroportadores basados en resultados suministrados por los bancos de sangre (10).

En nuestro hospital se realiza el despistaje obligatorio para el virus de la hepatitis B y C en los donantes de sangre y de órganos, los pacientes renales crónicos que ingresan a hemodiálisis, en los infectados por el virus del VIH, en este grupo se registraron pocos pacientes infectados con el virus VHB como el VHC, en las embarazadas está recomendado el despistaje de la infección por el VHB, pero no se implementa en forma obligatoria y en todos aquellos con sospecha de infección por este virus. En el servicio de gastroenterolología de nuestro hospital se registraron pocos pacientes con hepatitis $B$ crónica, así como por el virus $\mathrm{C}$, que se encuentran bajo tratamiento. 
El objetivo de este estudio es presentar dos pacientes con infección por hepatitis Bcrónica que acudieron al Hospital Nacional en setiembre del 2016 cuyo diagnóstico se realizó en forma accidental(IDAHS) y con probable transmisión vertical.

\section{PRESENTACIÓN DE LOS CASOS}

\section{Caso 1}

Mujer de 32 años, ama de casa, procedente de JA Saldívar. El 18 de noviembre del 2016 acude al Hospital Nacional por perdidas rojas, la paciente se encontraba cursando un embarazo de 36 semanas, siguió control prenatal en el centro de la seguridad social de su comunidad. Ella es internada para monitoreo fetal por oligoamnios, 24 hs después entra en trabajo de parto, la profesional médica que la asistió no tuvo tiempo de ponerse los guantes y entra en contacto con importante cantidad de sangre. La profesional que asistió el parto sin protección no tenía el esquema completo de vacunación para la hepatitis B y no contaba con anticuerpo protector, por lo que recibió inmunoglobulina en profilaxis y la vacunación para la hepatitis $\mathrm{B}$.

La paciente tenía como antecedentes el diagnóstico de hepatitis B en un control de rutina, realizado en Buenos Aires a la edad de 20 años. Recibió interferón por 6 meses y comprimidos cuyo nombre desconoce, se realizó una biopsia hepática cuyo resultado desconoce. Su pareja y el hijo de 14 años tienen el antígeno de superficie para hepatitis B (-). La paciente no tenía antecedentes de transfusión de sangre ni de ser consumidora de drogas ilícitas intravenosa. Refiere tener una hermana con hepatitis B, la madre tiene 70 años, tiene 12 hermanos desconociéndose el estado serológico para el VHB en el resto de sus hermanos. Los padres y hermanos se encuentran fuera así como en el interior del país.

El examen clínico es normal, se realiza los controles serológicos postparto en la paciente: La serología HIV (-), VHBC (-). El hepatograma es normal, HBsAg (+), Anti HBs (-), $\mathrm{HBeAg}<10 \mathrm{u}, \mathrm{HBeAg}(-)$,Anti-HBe Ac(+), HBcIgG (+), HBc AcIgM (-), hepatogramanormal.

La ecografía abdominal revela un hígado homogéneo, normal. Se interpreta el cuadro como una hepatitis B crónica inactiva. Se realiza dosaje de ADN viral para la conducta terapéutica.

El caso es interpretado como una hepatitis B crónica inactiva, por probable trasmisión vertical, se espera la carga viral para decidir biopsia hepática y tratamiento con tenofovir.

\section{Caso 2}

Paciente varón de 45 años, procedente de Pedro Juan Caballero, casado. Conocido portador de LES en tratamiento con inmunosupresor, consulta para realizarse estudios laboratoriales de rutina, ex tabaquista. Sin antecedentes de trasfusión de hemoderivados, niega ser consumidor de drogas ilícitas por vía intravenosa, se declara bebedor social.

Al examen físico como dato positivo de valor abdomen: blando, depresible, no doloroso. RHA presentes, signo de la onda liquida (-). Se palpa hepatomegalia a $4 \mathrm{~cm}$ del reborde costal derecho. Se constata punta de bazo. Ambos con superficie regular, no doloroso. Se solicita ecografía abdominal que revela hepatoesplenomegalia, sin nódulos ni zonas de fibrosis, liquido libre escaso interasas. Se solicita serología para Hepatitis B, por contar antecedentes de que la madre y 3 hermanos son portadores de hepatitis $\mathrm{B}$. La serología para hepatitis $\mathrm{C}(-), \mathrm{HBsAg}(+)$, HBcAgIgG (+), HBcAgIgM (-), HBeAg(-)yanti-HBe(+), carga viral: 113 copias.

El cuadro es interpretado como una hepatitis inactiva por probable trasmisión vertical por los antecedentes familiares. Se indica tratamiento con tenofovir por tratarse de un paciente con LES bajo tratamiento inmunosupresor por el riesgo de una reactivación virológica de la hepatitis B y se indica la realización de una biopsia hepática en proceso.

\section{DISCUSIÓN}

Los dos pacientes que se reportan en este estudio sufrieron probablemente una transmisión vertical de la infección por el VHB por los antecedentes familiares. En general, 10 a $20 \%$ de recién nacidos de madres $\mathrm{HBsAg}(+)$ y $90 \%$ de recién nacidos de madres $\mathrm{HBsAg} / \mathrm{HBeAg}(+)$ se infectan con el VHB (11).

La inmunización con gammaglobulina hiperinmune contra HVB (HBIG) y el iniciar la vacunación contra el VHB al momento del nacimiento reduce el riesgo de transmisión a menos del $10 \%$ en recién nacidos de madres HBsAg/HBeAg $(+)(11)$. 
En nuestro caso, la paciente era $\mathrm{HBeAg}(-)$, el recién nacido recibió la profilaxis postexposición con gammaglobulina hiperinmunepara hepatitis $\mathrm{B}$ y la primera dosis de la vacuna para hepatitis $B$.

Puesto que es alta la tasa de evolución hacia la cronicidad de los casos de hepatitis B adquirida en los primeros años de la vida ( $90 \%$ de los casos de transmisión perinatal, $50 \%$ en lactantes, $30 \%$ en niños menores de 3 años), se recomienda la detección de HBsAg en toda gestante al inicio del control prenatal, independientemente que la mujer haya sido vacunada o que tenga una prueba HBsAg previa, así como al momento del parto. La prueba debe ser realizada a toda gestante que no tenga una prueba de HBsAg previa durante el embarazo y en aquellas gestantes con riesgo alto de infección por VHB: usuarias de drogas parenterales, gestantes, que hubieran tenido más de una pareja sexual en los últimos 6 meses, gestantes cuyas parejas sean portadoras de HBsAg $(+)$ y aquellas que cursen 0 estén en tratamiento de una ITS y la vacunación infantil, que es una estrategia que permite reducir la prevalencia de portadores crónicos (10).

Los dos pacientes presentan una hepatitis B crónica inactiva por la interpretación de los marcadores serológicos y de la carga viral a títulos bajo. En ambos casos no se pudo estudiar el estado serológico del resto de la familia (hermanos, padres, algunos miembros de la familia viven en la Argentina y los otros en el interior del país). Se instó a los pacientes a que sus hermanos acudan a un puesto sanitario para el despistaje de la infección por el VHB.

Estos dos casos constituyen una llamada de atención sobre la trasmisión vertical del virus y la importancia de realizar el despistaje en la primera visita de la embarazada en el control prenatal.

La información disponible en el Paraguay sobre la prevalencia de las infecciones crónicas por el virus de la hepatitis B y C en la población es escasa. La prevalencia en gestantes según un estudio realizado por el Laboratorio Central de Salud Pública es de 0,379\% y en el mismo estudio en mujeres no gestantes fue levemente mayor 0,39\% (12). La muestra pudo estar sesgada debido a que la población incluye mujeres con pedido médico para un laboratorio público de referencia. Por otro lado, un estudio en donantes de sangre reportó una prevalencia de $0,41 \%$ (de $0,47 \%$ en el 2006 a 0,37\% en el 2011) (13). Se observaron prevalencias más altas en personas viviendo con el VIH (14), con una prevalencia global de 3,96\% (IC95\%: 3,03\%-5,06\%). Otros estudios realizados en poblaciones seleccionadas como en mujeres de un hospital psiquiátrico (15) y en una población de grupos étnicos no encontraron ninguna infección (16). En una población sesgada de pacientes con pedido médico, la prevalencia fue mayor $(4,5 \%)(17)$.

La vacunación para la hepatitis B está recomendada para los profesionales de blanco y es de distribución gratuita en nuestro país, hallándose disponible en todos los centros sanitarios. Sin embargo, la profesional a cargo de la paciente embarazada presentaba un esquema irregular e incompleto de la vacunación para la hepatitis B. Era de esperarse que no tuviera marcadores serológicos protectores, corriendo un riesgo de infección tras la exposición innecesario y prevenible con la vacunación. Así mismo, la paciente, a pesar de haber asistido en forma regular al control prenatal y contar con el antecedente de infección por el virus $B$, el médico que la atendió no solicitó ningún control serológico, exponiéndole al producto a una infección por trasmisión vertical. De no ser por el accidente laboral, el caso hubiera pasado desapercibido, lo cual revela un desconocimiento de los profesionales sanitarios sobre el manejo y prevención de esta infección. Ovando et al determinaron el nivel de cobertura vacunal para HVB en médicos residentes en el Hospital Central del Instituto de Previsión Social (18) y encontraron que solo el $66,6 \% \quad(n=102)$ de los 153 médicos tenían vacunación completa y $80(52,2 \%)$ refirieron accidente corto punzante. En otro estudio llevado a cabo por Irala y González para determinar la cobertura de vacunación del personal de salud del Hospital Materno Infantil Santísima Trinidad, considerando las vacunas provistas por el PAI, realizado durante el periodo 2008-2009 se encontró que la cobertura de vacunación del personal de salud del mencionado hospital fue deficitaria especialmente con la vacuna anti-hepatitis $B$, la cobertura global con esta vacuna fue solo de $23,7 \%$ (19).

También es necesario señalar que, a pesar de contar el hospital con un consultorio de salud laboral, se desconoce la cobertura vacunal contra la hepatitis B en los profesionales de la salud de nuestro hospital, situación que debe revertirse por las implicancias que conlleva la falta de cumplimiento del protocolo de vacunación y la posterior verificación de marcadores protectores contra esta infección en todos los profesionales de la salud, 
haciéndose énfasis en los profesionales con más riesgo de exposición como los cirujanos, los gineco-obstetras y todos aquellos con mayor manipulación de productos sanguíneos.

Es necesario un mejor conocimiento de la prevalencia de las hepatitis crónicas en la población de riesgo en nuestro país y de los obstáculos que pueden tener para acceder a los cuidados sanitarios necesarios para controlar y reducir las infecciones por el VHB. Las embarazadas, los niños, los profesionales de la salud, los infectados por el virus del VIH, los pacientes renales crónicos en hemodiálisis deberían considerarse el grupo prioritario de intervención y monitorización, con la serología para descartar la infección por el VHB, siendo primordial desarrollar campañas sanitarias dirigidas a estos grupos susceptibles con el fin de evitar la transmisión de este virus. Una de esas medidas corresponde al desarrollo de la vacuna recombinante. El $92 \%$ de los países en el mundo ha implementado esta vacuna con una cobertura global de $69 \%$ (3), desconociéndose su impacto en los grupos de riesgo en nuestro país.

\section{REFERENCIAS BIBLIOGRÁFICAS}

1. Calderón E, Yang Lai R, Calero Bernal M, Martínez Rísquez MT, Calderón $M$, de la Horra Padilla C. Hepatitis crónicas virales en población inmigrante en España. Rev Esp Salud Pública. 2014; 88:811-8.

2. Dixit VK, Kumar S. Incidentally detected asymptomatichbsag positive subjects. http://www.hepatitisbannual.org

3. Quintana Duque M, Regino W, Concha Mejía A.Hepatitis B Inactiva. Decisiones en la práctica clínica. 2009 Asociaciones Colombianas de Gastroenterología, Endoscopia digestiva, Coloproctología y Hepatología Hepatitis B Annual, Year 2008;5(1):95-101.

4. Jaramillo C, Navas MC. Variantes de escape del virus de la hepatitis B.Rev Chilena Infectol. 2015;32(2):190-7.

5. Lok A. Clinical manifestations and natural history of hepatitis B virus infection. Upto date.

6. Barahona García R, Hevia Urrutia F, Madrigal-Borloz M. Concenso de manejo de HBV En Costa Rica. AMC. 2008;50(Supl3). Disponible en: http://actamedica.medicos.cr/index.php/A cta_Medica/article/viewFile/462/434

7. Lok A. Overview of the management of hepatitis $B$ and case examples. Up date

8. Jaramillo T. Artículo de revisión: hepatitis viral B y su manejo. Rev. Med. FCMUCSG. 2010;16(4):307-32.

9. Dirección General de Vigilancia de la Salud. Ministerio de Salud Pública y Bienestar Social. Guía Nacional de Vigilancia y Control de Enfermedades. Sistema Nacional de Vigilancia Epidemiológica. Paraguay, 2015. Disponible en: http://www.vigisalud.gov.py/documentos/ Guia-Vigilancia-2015.pdf

10. Organización Mundial de la Salud, Organización Panamericana de la Salud. Salud en las Américas. Washington, D.C: OMS/OPS; 2012. Publicación Científica y Técnica No 204.

11. Cabezas-Sánchez C, Donayre F. Gestación e infección por el virus hepatitis B. Rev Per Ginecol Obstet. 2010;183-92.
12. Olmedo GB, Zorrilla $M$, Bobadilla $M L$, Villagra $V$, Avalos DS, Huber $C$ et al. Serorreactividad al antígeno de superficie del virus de la Hepatitis $B$ en mujeres que acudieron al Laboratorio Central de Salud Pública. Asunción, Paraguay. Mem. Inst. Investig. Cienc. Salud. 2015;13(3):96

13. Marquez Roa NA, Lemir de Zelada MO, Molas AC. Frecuencia serológica de infección por Trypanosoma cruzi en donantes de sangre en el Paraguay entre los años 2006 y 2011. Mem. Inst. Investig. Cienc. Salud. 2013;11(2):26-31.

14. López G, Aguilar G, Ovelar P, Samudio T, Valdez MR, Aguayo N. Prevalencia de Hepatitis B (VHB) y Hepatitis C (VHC) en Personas que Viven con el VIH/SIDA (PVVS).Rev. Inst. Med. Trop. 2011;6(Suplemento).

15. Rovira C, Picagua E, Martínez C, Ferreira L, Granado E, Giménez V et al. Hepatitis B: antígeno de superficie y anticuerpos post vacunales en mujeres del Hospital Psiquiátrico de Asunción, Paraguay. Mem. Inst. Investig. Cienc. Salud. 2011;9(2):57-63.

16. Rovira C, Monzón I, Almirón M. Hepatitis virales en diferentes grupos étnicos del Paraguay. Memorias del Instituto de Investigaciones en Ciencias de la Salud. 2005;3(1):5-8.

17. Rovira $C$, Picaguá $E$, Ferreira L, Giménez V, Carpinelli MM, Granado E. Prevalencia de marcadores serológicos de hepatitis virales en una población seleccionada: Experiencia de un servicio universitario. Años 2000-2007. Memorias del Instituto de Investigaciones en Ciencias de la Salud. 2009;7(1):20-6.

18. Ovando F, Guerrero A, Olmedo R. Vacunación contra Hepatitis $B$ en una población de riesgo. Rev. Salud Pública Parag. 2013;3(1):36-40.

19. Irala Cabrera S, González MM. Cobertura de vacunación del personal sanitario del Hospital Santísima Trinidad, AsunciónParaguay. Rev. Salud Pública Parag. $2011 ; 1(2): 4-10$. 